\title{
A modified bilobed flap design for nasal tip defects
}

\author{
Zhi-Guo Wang ${ }^{1}$, Xiu-Juan Chen ${ }^{2}$, Zhen-Yu Chen ${ }^{1}$ \\ ${ }^{1}$ Department of Burn and Plastic Surgery, Affiliated Hospital of Qingdao University, Qingdao 266003, Shandong, China. \\ ${ }^{2}$ Department of Stomatology, Affiliated Hospital of Qingdao University, Qingdao 266003, Shandong, China.
}

Address for correspondence: Dr. Zhen-Yu Chen, Department of Burn and Plastic Surgery, Affiliated Hospital of Qingdao University, No.16 Jiangsu Road, Shinan District, Qingdao 266003, Shandong, China. E-mail: wzg_qd@126.com

\begin{abstract}
ABSTRAC
Aim: The correction of nasal tip defects presents many challenges. Zitelli's bilobed flap has been widely used for such repairing defects, but may be complicated by interrupted scars on the nasal dorsum. Our study evaluates the design principles, results, and advantages of a modified bilobed flap for repairing nasal tip defects. Methods: The primary lobe was located between the defect and the cheek, and the second lobe was located in the cheek. The width of the primary lobe was equal to that of the primary defect. The length of the primary lobe was $10 \%$ longer than the distance of the distal defect edge to the pivot point of the flap. The length of the second lobe was $30 \%$ longer than the distance of the distal defect edge to the pivot point of the flap. The width of the second lobe was $90-100 \%$ of that of the primary lobe. The ability to close the defect under minimal tension, the cosmetic appearance, and any complications were evaluated. Results: This technique was performed in 34 cases; defect size ranged from $0.8 \mathrm{~cm} \times 0.9 \mathrm{~cm}$ to $1.2 \mathrm{~cm} \times 1.8 \mathrm{~cm}$. All defects were closed under minimal wound tension, all scars were inconspicuous, no obvious complications occurred, and the aesthetic outcomes were considered favorable. Conclusion: The modified bilobed flap can provide satisfying outcomes with lower morbidity and inconspicuous scarring. It is simple and suitable for repairing small- to medium-sized defects in the nasal tip.
\end{abstract}

Key words:

Bilobed flap, nasal reconstruction, nasal tip defect

\section{INTRODUCTION}

Resection of benign lesions is a common cause of nasal defects in young patients. The conventional bilobed flap (Zitelli's design) used for reconstruction of defects of the lower third of the nose traditionally utilizes skin from the mid dorsum and the sidewall of the nose. ${ }^{[1]}$ However, the conspicuous scarring and loss of conformity in the cosmetic subunits of the nose might be unacceptable to young patients, particularly in individuals with darker skin tones. Herein, we describe a modified bilobed flap that utilizes skin from

\begin{tabular}{|l|l|}
\hline \multicolumn{2}{|c|}{ Access this article online } \\
\hline Quick Response Code: & Website: \\
\hline & www.parjournal.net \\
\cline { 2 - 3 } & \\
\hline
\end{tabular}

the nose and cheek, and review the advantages of this technique's use in nasal tip reconstruction.

\section{METHODS}

Our study comprised 34 patients who had defects of the nasal tip. We conducted a retrospective review of all patients who had undergone defect reconstruction with the modified bilobed flap by a single surgeon at the Department of Plastic Surgery, Affiliated Hospital of Medical College, Qingdao University, from July 2008 to July 2013. The review board of our Affiliated Hospital approved this study and all patients involved in this article agreed to have their pictures published and signed the consent form. Outcome measures included the ability to close the defect with minimal tension, cosmetic appearance, any complications, and any need for further repair. 


\section{Flap design}

Based on the designs of Zitelli and Baker, ${ }^{[1]}$ Xue et al. ${ }^{[2]}$ planned a superiorly based bilobed flap obtained from the nasolabial region. First, two arcs were marked to define the boundaries of the flap and its proper angulations. The radius and diameter of the nasal defect were measured with calipers. A pivot point was placed in the nasal sidewall and was located one radius from the free edge of the defect. The center line of the defect was marked. The radius of the center arc was equal to the diameter of the nasal defect. The radius of the distal arc was equal to the distance between the distal edge of the defect and the pivot point. Based on the pivot point, the two arcs were marked with a standard geometry compass.

Second, the two lobes of the flap were configured. The primary lobe was located between the defect and the cheek and was slightly larger than the primary defect. The width of the primary lobe was equal to that of the primary defect. The length of the primary lobe was $10 \%$ longer than the distance of the distal defect edge to the pivot point of the flap. The second lobe was located in the cheek and was slightly smaller than the primary lobe. The length of the second lobe was $30 \%$ longer than the distance of the distal defect edge to the pivot point of the flap. The width of the second lobe was $90-100 \%$ of that of the primary lobe. The two flaps rotated a total of $90^{\circ}-100^{\circ}$. Based on the two arcs, the two lobes of the flap were marked.

\section{Surgical technique}

The lesions were removed below the nasal superficial musculoaponeurotic system. The specimens were sent for histopathological examination to ensure clearance of the margins.

Incisions were made along the previously described markings. The primary lobe was undermined above the perichondrium of the nasal cartilage to promote adequate tissue perfusion of the flap. Once this layer was reached, the flap was easily elevated. The second lobe was elevated in the plane of the superficial fascia, and the pedicle portion was separated with blunt dissection to preserve the blood supply to the deep tissue. It is important that the thickness of the primary lobe is equal to that of the second lobe.

The two lobes were transposed to their desired locations. The primary and secondary defects were repaired by rotation of the two lobes. Hemostasis was performed. A 5-0 absorbable monofilament suture was used to close the deep layer, which allowed the skin layer to be closed under minimal tension. The redundant distal portions of the lobes were removed with regard to the state of the closure tension. The tertiary wound was closed directly in a side-to-side manner. A 6-0 nylon suture was used to close the skin layer. Rubber drainage was applied to prevent subcutaneous hemorrhage and was removed 1 day later. The stitches were removed from the skin 5-7 days later [Figures 1 and 2].

\section{RESULTS}

Reconstruction procedures using the modified bilobed flaps were performed in 34 cases with tissue defects of the nasal tip. The study sample included 5 male and 29 female patients, with an average age of $27.14 \pm 7.79$ years. Twenty patients presented with nevus, 8 with granuloma, and 6 with fibroma. The size of the defects ranged from $0.8 \mathrm{~cm} \times 0.9 \mathrm{~cm}$ to $1.2 \mathrm{~cm} \times 1.8 \mathrm{~cm}$. All defects were closed successfully without difficulty using this technique. The defects were repaired under minimal closure tension as a single-stage procedure. The patients were then followed for 3 months and 18 months. No severe complications were found to have occurred after any of the operations. There were no dissymmetry deformities or retraction deformities of the nasal ala in any of the cases.

We did not find any patient with nasal valve collapse,

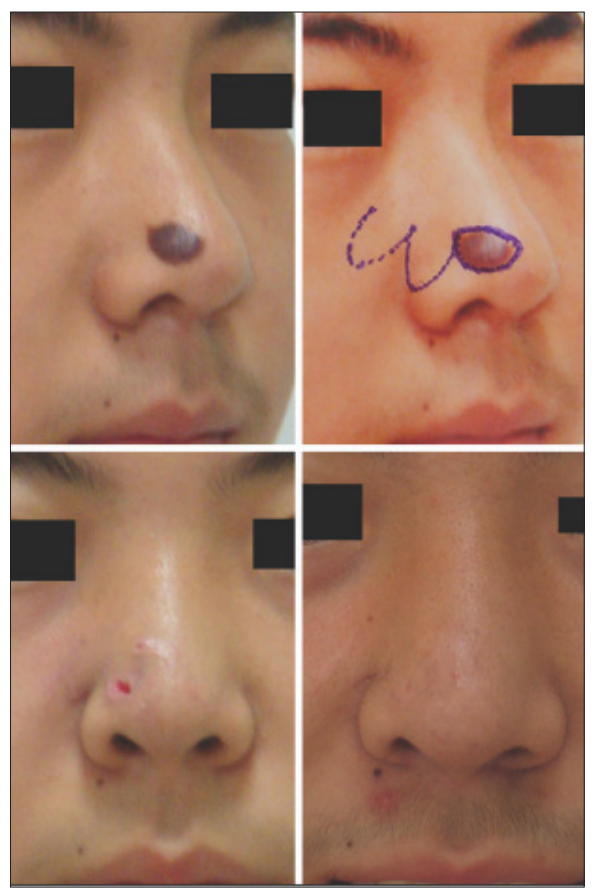

Figure 1: Modified bilobed flap reconstruction of a nasal tip defect in a 19-year-old man: before the operation (upper left); the bilobed flap design (upper right); incision infection 1 week after the operation (lower left); at the 13 months follow-up (lower right)

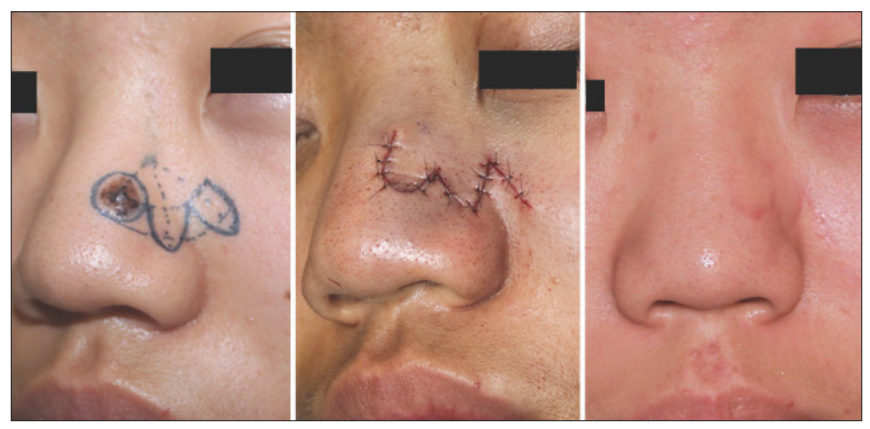

Figure 2: Modified bilobed flap reconstruction of nasal tip defect in a 20-year-old woman: the bilobed flap design (left); immediately after the operation (center); at the 1-year follow-up (right) 
and no patients complained of ventilation barriers. Three patients presented with an obscure alar crease and did not require further repair. There was incision infection in one patient 3 days after the operation owing to the exudation of a sebaceous gland. The wound healed well 10 days later [Figure 1]. There was mild trapdoor deformity in one patient 1 month after the operation; however, the deformity disappeared after two steroid injections. All of the flaps survived completely with ideal color and texture matching. All of the scars healed well and were inconspicuous. All 34 patients were satisfied with the results.

\section{DISCUSSION}

Before the techniques for nasal reconstruction being discussed, some general comments regarding the nature of nasal skin are warranted. The nasal skin is typically divided into three zones. ${ }^{[3]}$ Zone I, the upper half of the nose, is the thin, loose, compliant, and non-sebaceous skin of the dorsum and sidewalls. In contrast, Zone II includes the thick, taut, non-compliant, and sebaceous skin of the nasal supratip, tip, and alae. It readily reforms to its previous shape, and thus it is difficult to contour and reconstruct. Due the thickness and stiffness of the sebaceous skin, even small defects cannot be closed in a linear manner, as the surgical tension might cause obvious nasal deformation. Zone III encompasses the areas of the triangles, columella, and nasal infratip lobule, where the skin again becomes thin, loose, and nonsebaceous. Consideration of such categories is critical in the nasal reconstruction of small- to medium-sized defects as the type, color, and texture of the skin surrounding the nasal defect play a critical role in ultimately determining the optimal method for reconstruction.

In conventional algorithms for nasal reconstruction, classic options for the repair of defects in Zone II include the bilobed flap, nasolabial flap, dorsal nasal flap, forehead flap, and full-thickness skin graft. These reconstruction options are time-tested and reliable, although none is without disadvantages.

The nasolabial flap is a good reconstructive option for lateral nasal defects such as defects in the sidewall and alae. The transposition design can be performed as a single-stage procedure, and can provide excellent color and texture matching. However, for central defects in the nasal supratip and tip, an interpolated design should be performed. The pedicle has to be divided 3 weeks later, and the flap has to be reshaped 2-3 months later.

The bilobed flap is also known as the "workhorse flap" for the reconstruction of defects of the lower third of the nose. ${ }^{[1]}$ It was first described by the Dutch surgeon Esser in 1918. ${ }^{[4]}$ Esser's initial flap design described two equally sized transposition flaps transferred to cover defects of the distal nasal tip. The primary lobe transposes $90^{\circ}$ to cover the primary tissue defect. The second lobe also transposes $90^{\circ}$, to cover the surgical defect produced by the displacement of the primary lobe. The primary lobe is located on the nasal dorsum adjacent to the primary defect. The second lobe is located on the more proximal nasal dorsum. Due to the large transposition arc, the flap rotation has to be accomplished with significant surgical effort and high wound-closure tension. It is inevitable that numerous significant standing deformities are produced after this operation, including "dog ear", alar displacement, and alar asymmetry. A second repair procedure is necessary for some patients.

In 1989, Zitelli $^{[5]}$ modified the bilobed flap to include a total transposition arc of $90^{\circ}-110^{\circ}$ and an approximately $45^{\circ}$ pivotal arc between each lobe. Zitelli's bilobed flap uses skin from the mid dorsum and the sidewall. Smaller angles of flap transposition produce less severe "dog ear" deformities along the border of the flap. Furthermore, smaller angles of flap transposition allow the surgeon to transfer the flap more easily. The flap can be rotated with lower wound tension, which produces less alar displacement. This design clearly decreases the likelihood of complications. It is especially suitable for the reconstruction of defects of the lower third of the nose.

However, vertical scarring on the mid dorsum and interrupted scarring on the tip occurs after using Zitelli's bilobed flap. The wound might heal well and be inconspicuous in older patients or patients with compliant nasal skin. However, the scar can be conspicuous in younger patients, particularly in individuals with darker skin tones. Most of the patients in our study could not accept the predicted outcomes of using Zitelli's bilobed flap. A modified bilobed flap utilizing skin from the lower dorsum and cheek was therefore recommended to these patients, and they agreed with the plan.

This technique combines the advantages of the nasolabial flap and Zitelli's bilobed flap, and thus offers more advantages. First, it is a single-stage procedure and is less technically complex. The two flaps (the primary and secondary lobes) are transposed with a total arc no more than $90^{\circ}-100^{\circ}$. The flaps can be rotated easily due to the smaller angles. Although the skin beside the defect is thick and noncompliant, the primary lobe can be transposed to the correct location with small closure tension. The surgeon can repair the second defect easily with a nasolabial flap (the second lobe).

Second, this technique has lower morbidity. Any degree of alar asymmetry can be quite noticeable since the nose is the most prominent part of the center of the face. Accordingly, one of the most important principles of nasal reconstruction is that asymmetry deformity should be avoided. As described above, the skin on the nasal dorsum and sidewall is thin, loose, compliant, and 
nonsebaceous, and the skin on the distal nose is thick, taut, noncomplaint, and sebaceous. Improper wound tension could not only lead to hypertrophic scarring, but also alar displacement. Therefore, it is not recommended that defects of distal nose be treated primarily with adjacent undermining when the defect is small. The surgeon must be acutely aware of the influence of the size of the lobes when using the bilobed flap, as both the width and the length of the lobes can affect the final incomes of reconstruction.

Moy et $a l .{ }^{[6]}$ suggested that the diameter of the primary lobe should be $90-100 \%$ of that of the primary defect and that the diameter of the second lobe should be $80-85 \%$ of that of the primary lobe. In our study, the diameter of the primary lobe was equal to that of the primary defect, which can ensure lower closure tension for repair of the primary defect. Alar displacement may result if the diameter of the primary lobe is even slightly smaller. In contrast to techniques described in previous reports, in our study, the second lobe was taken from the cheek and had a diameter of $90-100 \%$ of that of the primary lobe. In fact, the second lobe is a nasolabial flap. The size of the second lobe is chosen on a case-by-case basis. The cheek has more abundant and lax skin than the nasal sidewall; therefore, the cheek can provide a larger flap than can the nasal sidewall. Tertiary defects on the cheek can also be more easily closed in a linear manner.

In 1987, Dzubow discussed the effect of pivotal restraint on flap rotation and transposition. ${ }^{[7]}$ He stated that the flap was restrained by the tissue located around the pivotal point (the base of the flap), when any flap of tissue was either rotated or transposed around a pivotal point. Thus, the bilobed flap is shortened after it is transposed to a new site. The greater degree of flap movement around the pivotal point, the more the flap shortens.

The main reported disadvantage of Zitelli's bilobed flap is alar retraction resulted from distal flap tension. Cho and Kim stated that this distal tissue retraction and distortion is a result of pivotal restraint..$^{[8]}$ The rotation of the bilobed flap causes the flap to shorten, thereby creating a gap that must be spanned by the distal edge of the defect. Their study in human cadavers demonstrated that lengthening the primary lobe in Zitelli's design could compensate for this expected gap and allow for less tension at the distal wound edge. Such a closure would prevent distal retraction and reduce anatomic distal distortion.

Given this concept of pivotal restraint, the loss of flap length must be accounted for during the bilobed flap design. Some researchers proposed to lengthen the flap. ${ }^{[2,7-9]}$ In our study, the length of the primary lobe was slightly longer than the distance of the distal defect edge to the pivot point of the flap, and the length of the second lobe was slightly longer than that of the primary lobe. The redundant distal portions of the flaps are removed as needed after the two lobes are transposed into their desired locations. Neither undersized nor oversized flaps are recommended as the former might lead to alar elevation, while the latter can push the alar rim inferiorly and result in trapdoor deformation. The ideally sized lobe of the bilobed flap should neatly cover the defect under minimal closure tension.

Third, the scars are less obvious. As already noted, the nose is the most prominent part of the face; therefore, interrupted scars on their nose might not be acceptable to many younger patients in Asia. ${ }^{[10]}$ Mature scars are more visible in individuals with darker skin tones than in those with lighter skin tones. Surgeons should therefore aim to decrease the number of scars and control hypertrophic scars on the nose. In contrast to previous designs, there were no noticeable scars on the mid-dorsum in patients in our study. Most incisions were placed in the natural lines such as the alar crease and the nasolabial groove. As described above, all defects were closed under minimal tension; therefore, less hypertrophic scarring occurred.

Lastly, this technique can provide better texture matching than is available through the conventional bilobed flap. The skin of the distal nose is thick and dense with sebaceous glands, similar to the skin of the nasolabial region. In contrast, the skin of the nasal dorsum and sidewall is thin and non-sebaceous. Particular attention should be paid to ensuring that the exudation of the sebaceous glands is removed in time to prevent incision infection.

The disadvantage of our design is that the primary lobe might destroy the anatomy of the alar crease and result in an obscure alar crease-a complication that can be avoided through careful suturing. In addition, the technique is only suitable for small- to medium-sized defect repair, due to the limited size of the primary lobe.

In conclusion, the modified bilobed flap can provide satisfying outcomes with lower morbidity and inconspicuous scarring. It is simple and suitable for repairing small- to medium-sized defects in the particular area of nasal tip.

\section{Financial support and sponsorship}

Nil.

\section{Conficts of interest}

None declared.

\section{REFERENCES}

I. Zitelli JA, Baker SR. Bilobe flaps. In: Baker SR, Swanson NA, editors. Local Flaps in Facial Reconstruction. St. Louis, MO: Mosby; 1995. p. 165-80.

2. Xue CY, Li L, Guo LL, Li JH, Xing X.The bilobed flap for reconstruction of distal nasal defect in Asians. Aesthetic Plast Surg 2009;33:600-4.

3. Burget GC, Menick FJ. Repair of small surface defects. In: Burget GC, Menick FJ, editors. Aesthetic Reconstruction of the Nose. St. Louis, MO: Mosby; 1994. p. II7-56.

4. Esser JF. Gestielteloakle Nasenplastikmitzweizipfl en Lappen, Deckung 
des sekundaren Defektesvomersten Zipfeldurch den Zweiten. Deutsche Zeitschriftür Chirurgie 1918;143:335-9.

5. Zitelli JA. The bilobed flap for nasal reconstruction. Arch Dermatol 1989; 125:957-9.

6. Moy RL, Grossfeld JS, Baum M, Rivlin D, Eremia S. Reconstruction of the nose utilizing a bilobed flap. Int J Dermatol 1994;33:657-60.

7. Dzubow LM.The dynamics of flap movement: effect of pivotal restraint on flap rotation and transposition. J Dermatol Surg Oncol 1987; I 3:1348-53.

8. Cho M, Kim DW. Modification of the Zitelli bilobed flap: a comparison of flap dynamics in human cadavers. Arch Facial Plast Surg 2006;8:404-9.

9. Cook JL.A review of the bilobed flap's design with particular emphasis on the minimization of alar displacement. Dermatol Surg 2000;26:354-62.
10. Zhi-Guo W, Quan-Chen X, Rui-Xia K, Zhen-Yu C, Ran H. Principles of hatchet-skin flap for repair of tissue defects on the cheek. Aesthetic Plast Surg 2012;36:163-8.

How to cite this article: Wang ZG, Chen XJ, Chen ZY. A modified bilobed flap design for nasal tip defects. Plast Aesthet Res 2014;1:16-20.

Received: 07-05-2014; Accepted: 29-05-2014 